\title{
Giant sigmoid diverticulum: clinical and radiological features
}

\author{
D. R. FOSTER AND B. ROSS ${ }^{1}$
}

From the Department of Radiology, Northern General Hospital, Sheffield

SUMMARY Two case reports of giant sigmoid diverticulum associated with diverticular disease of the sigmoid colon are presented. The clinical and radiological features of 30 similar cases found in the literature are reviewed. Our two cases represent the largest recorded diverticulum and the oldest recorded patient with this condition.

Diverticular disease of the colon is a common clinical finding. Its incidence on barium enema or post-mortem examination rises with increasing age so that at the age of 60 years it is found in at least $30 \%$ of patients studied. Giant sigmoid diverticulum is a rare complication and only 30 cases have been described in the world literature since it was first reported by Hughes and Greene in 1953. Only one report of two cases (Johns and Hartley, 1976) has appeared in the British literature. Confirmation was obtained at operation in both our cases, which illustrate the typical radiological appearances.

\section{Case 1}

A 61-year-old female was admitted with a three week history of central abdominal pain. This had become severe and persistent over the previous 12 hours and was accompanied by nausea and occasional vomiting.

On clinical examination she appeared pale and unwell. There was considerable abdominal distension but bowel sounds were normal.

Haematological and biochemical investigations were normal. Chest radiograph was normal. Erect and supine abdominal films showed a large gasfilled cyst occupying the greater part of the abdomen (Fig. 1), and containing a fluid level on the erect film (Fig. 2). The lesion measured $29 \mathrm{~cm}$ in diameter on the radiographs.

At laparotomy a large air-containing sac arising from the sigmoid colon was found. The cyst was adherent anteriorly to the uterus and bladder. The cyst was excised together with the uterus and a cuff of sigmoid colon. A defunctioning colostomy was performed.

The patient made an uneventful recovery and the

'Present address: Department of Radiology, Royal Hospital, Sheffield.

Received for publication 11 May 1977

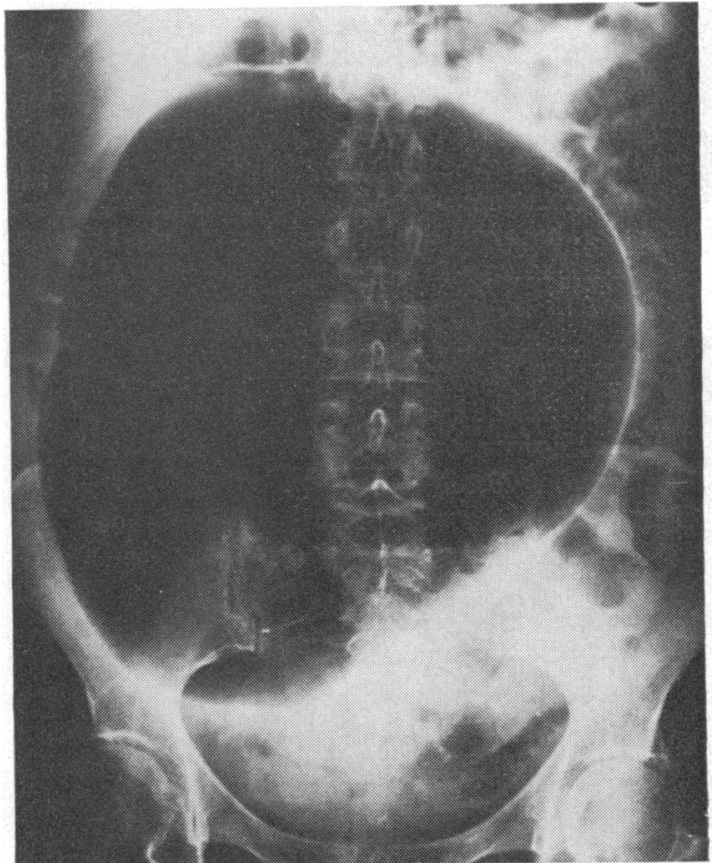

Fig. 1 Supine abdominal radiograph showing large gasfilled cyst.

colostomy was closed two months later. A barium enema before this showed occasional diverticula in the sigmoid colon but no other abnormality.

\section{PATHOLOGICAL REPORT}

The specimen consisted of a massive sac lined by a thick white gelatinous membrane. It contained serous fluid but no communication with the colon could be demonstrated. Histology showed the lining to consist of chronic inflammatory cells. There was no evidence of malignancy. 


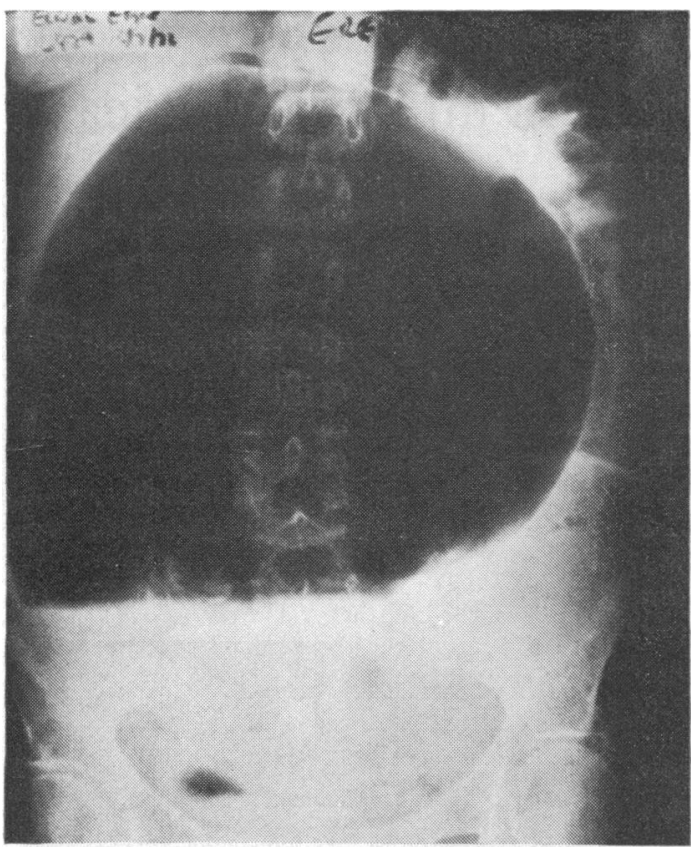

Fig. 2 Erect abdominal radiograph showing fluid level.

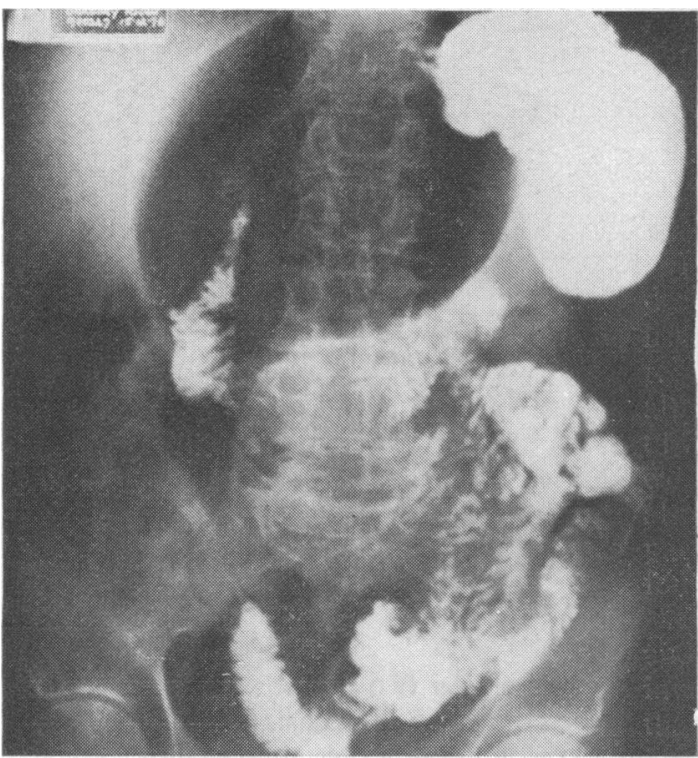

Fig. 3 Barium meal and follow-through showing large cyst displacing stomach.

\section{Case 2}

An 86-year-old man was admitted for investigation of an epigastric mass. He gave a six week history of epigastric pain accompanied by the passage of fresh blood per rectum.

Clinical examination revealed a fit man with a large soft epigastric mass.

Haematological and biochemical investigations were normal. Chest radiograph was also normal. Barium meal and follow-through revealed a large gas filled structure measuring $17 \mathrm{~cm}$ in diameter lying in the epigastrium and displacing the stomach (Fig. 3). A barium enema performed subsequently showed diverticular disease of the sigmoid colon with a large solitary diverticulum arising from its superior margin (Fig. 4). Barium was demonstrated within the diverticulum. A radiological diagnosis of giant sigmoid diverticulum was made.

At laparotomy a large cyst was found at the apex of the sigmoid loop with marked diverticular disease of the sigmoid colon. Sigmoid colectomy with endto-end anastomosis was performed. The patient made an uneventful recovery after operation.

\section{PATHOLOGICAL REPORT}

The specimen consisted of a $21 \mathrm{~cm}$ length of large bowel with attached massive diverticulum measuring $14 \times 10 \times 10 \mathrm{~cm}$. The mucosa appeared intact. The junction of the bowel and diverticulum appeared occluded. The diverticulum showed an acute chronic inflammatory change in its wall. There was no evidence of malignancy.

\section{Discussion}

In 1953, Hughes and Greene first described a case of 'solitary air cyst' arising from the antimesenteric

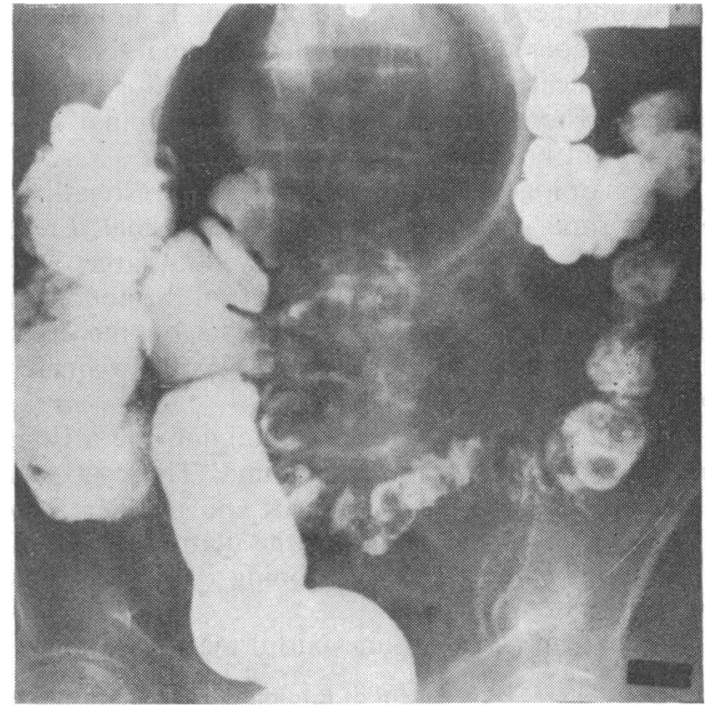

Fig. 4 Barium enema showing barium within the cyst and associated diverticular disease of the sigmoid colon. 
border of the sigmoid colon. Since then, 30 cases of giant sigmoid diverticulum or giant gas-filled cyst of the sigmoid colon have appeared in the literature.

The age of these patients ranges from 38 to 78 years. Our patient of 86 years is the oldest recorded patient with a giant sigmoid diverticulum. The sex distribution is equal. All cases of giant colonic diverticula have occurred in the sigmoid colon with the exception of one, which occurred in the transverse colon (Sagar, 1973). Plain abdominal radiographs reveal a lucent cyst-like structure varying in size from 6 to $25 \mathrm{~cm}$ or more in diameter, although that in case 1 measured $29 \mathrm{~cm}$ and is the largest sigmoid diverticulum ever reported. The diverticulum lies anteriorly in close relationship with the sigmoid colon and may displace normal intra-abdominal structures. The presence of an air/fluid level as in case 1 has been described in 12 of the 30 cases reported. Barium enema examination has been performed in the majority of cases. Communication between the diverticulum and sigmoid colon is demonstrated in approximately $25 \%$ of cases (Moss, 1975). Associated colonic diverticulosis has been noted in all reported cases with one exception (Vanapruks and Fuhrman, 1969).

The clinical presentation is variable. Both our patients presented with abdominal pain of several weeks' duration, while one also complained of rectal bleeding. These features correspond closely with those described by Rabinowitz et al. (1974) in their series of five cases. Nausea, vomiting, and fever may also occur. Complications have occurred in two cases-namely, perforation (Macbeth and Riddle, 1964) and volvulus (Silberman and Thorner, 1961).

The treatment of choice is sigmoid colectomy with primary anastomosis. When examined histologically the cysts are up to $1 \mathrm{~cm}$ in thickness and are composed of granulation and fibrous tissue containing chronic inflammatory cells. There is usually a lack of both mucosal lining and muscular elements in their walls suggesting an acquired, rather than a duplication origin (Bergeron and Hanley, 1965). The gas contained in a cyst was analysed by Sibson and Edwards (1972). They postulated that bacteria played a significant role in the production of gas.

The differential diagnosis of a large gas-filled structure in the abdomen is limited. Volvulus of the caecum or sigmoid colon can result in this appearance but will be associated with signs of intestinal obstruction. Enteric duplication cysts occur in relation to the mesenteric side of the bowel and commonly present in childhood. In contrast with a giant sigmoid diverticulum, the wall of a duplication contains smooth muscle bundles. In pneumatosis cystoides intestinalis, cysts may be found in the small bowel or colon but they are always smaller in size and multiple.

The gall bladder may occasionally contain large amounts of air. This is most commonly caused by a fistulous communication with the gastrointestinal tract either due to a perforating gall stone or peptic ulcer. The clinical history, together with the presence of air in the biliary tree, enables this condition to be readily distinguished. Less commonly, the presence of gas-forming organisms in the gall bladder may result in emphysematous cholecystitis, a disease typically found in elderly diabetic patients.

Giant duodenal diverticulum (Millard et al., 1974) and a large cystic Meckel's diverticulum (Lerner et al., 1953) have also been described but may be excluded by their relation to the duodenum and ileum respectively.

The radiological finding of a large lucent systic structure related to the sigmoid colon in an elderly patient is suggestive of a giant sigmoid diverticulum. Confirmatory evidence is obtained by performing a barium enema examination, while the treatment of choice is elective surgical removal.

We wish to thank the surgeons of the Northern General Hospital for permission to report on their cases and Dr P. Grech for his valuable advice.

\section{References}

Bergeron, R. B., and Hanley, P. H. (1955). Giant sigmoid diverticulum. American Journal of Surgery, 109, 660-662.

Hughes, W. L., and Greene, R. C. (1953). Solitary air cyst of peritoneal cavity. Archives of Surgery, 67, 931-936.

Johns, E. R., and Hartley, M. G. (1976). Giant gas-filled cysts of the sigmoid colon: a report of two cases. British Journal of Radiology, 49, 930-931.

Lerner, H. H., Levenson, S. S., and Kateman, A. E. (1953). Meckel's diverticulum: a new roentgen diagnostic sign and case report. American Journal of Roentgenology, 69, 268-271.

Macbeth, W. A. A. C., and Riddle, P. R. (1964). Gas-filled abscess cavity as manifestation of diverticulitis of the colon. British Journal of Radiology, 37, 861-862.

Millard, S. R., Ziter, M. H., and Slover, W. P. (1974). Giant duodenal diverticula. American Journal of Roentgenology, 121, 334-337.

Moss, A. A. (1975). Giant sigmoid diverticulum. Digestive Diseases, 20, 676-683.

Rabinowitz, S. G., Farman, S., Dallemand, S., Twerskey, S., and Rosen, Y. (1974). Giant sigmoid diverticulum. American Journal of Radiology, 121, 338-343.

Sagar, S. (1973). Giant solitary diverticulum of transverse colon with diverticulosis. British Journal of Clinical Practice, 27, 145-146.

Sibson, D. E., and Edwards, A. S. (1972). Giant gas-filled cysts of sigmoid colon. Postgraduate Medical Journal, 48, 180-184.

Silberman, E. L., and Thorner, M. C. (1961). Volvulus of giant sigmoidal diverticulum. Journal of the American Medical Association, 177, 782-784.

Vanapruks, S., and Fuhrman, M. (1969). Giant solitary gas cyst of the sigmoid colon. Radiology, 92, 1553-1554. 